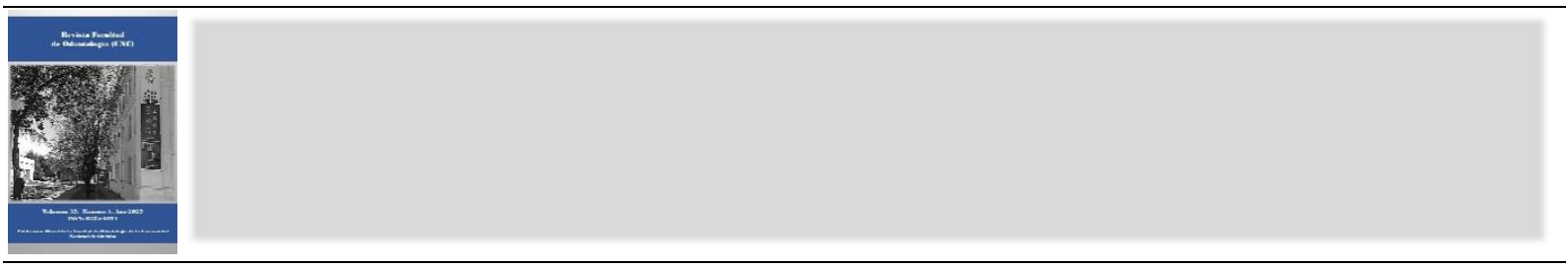

Caso Clínico/ Clinical Case

\title{
Tatuaje por amalgama: Caso Clínico
}

\section{Amalgam Tattoo: Clinical Case}

Bonal-Medina Alma A ${ }^{1}$, Carrillo-García Nicol Y ${ }^{1}$, Riggen-Martínez Guillermo E², Gutiérrez-Cortés Evangelina ${ }^{3}$, Valdivia Andréa Dolores ${ }^{4 *}$

\footnotetext{
${ }^{1}$ Universidad Autónoma de Guadalajara, Facultad de Odontología, Departamento de Odontopediatria, Universidad Autónoma de Guadalajara

${ }^{2}$ Universidad Autónoma de Guadalajara, Facultad de Odontología, Departamento de Cirugia Oral, Universidad Autónoma de Guadalajara ${ }^{3}$ Universidad Autónoma de Guadalajara, Facultad de Odontología, Departamento de Patología Bucal, Universidad Autónoma de Guadalajara.

${ }^{4}$ Universidad Autónoma de Guadalajara, Facultad de Odontología, Universidad Autónoma de Guadalajara, Departamento de Odontologia Especializada
}

*Correspondencia a/Corresponding to:

Andréa Dolores Correia Miranda Valdivia DDS, MSc, PhD*

Facultad de Odontología, Departamento de Odontologia Especializada,

Universidad Autónoma de Guadalajara. Guadalajara, Jalisco, MÉXICO

Calle Escorza, \#526, Colonia Moderna. CP. 44150

Teléfono: +52 (33) 1503-3937

Correo electrónico/E-mail: andrea.correia@edu.uag.mx

Rev Fac Odont (UNC). 2022; 32 (1): 37-45

doi: 10.25014/revfacodont271.2020.32.1.37

https://revistas.unc.edu.ar/index.php/RevFacOdonto

Received 13 July 2021; Revised 23 February 2022; Accepted 28 February 2022

\begin{abstract}
The amalgam tattoo or focal argyrosis, is an iatrogenic lesion that follows the traumatic implantation of amalgam particles in soft tissue or the passive transfer by chronic friction of the mucosa against an amalgam restoration is characterized by the deposit of remains of restorative material composed of a mixture of silver, mercury, zinc, tin and copper in the connective tissue. Objective to present the clinical case of a patient with amalgam tattoo and the treatment that was carried out prior to his dental rehabilitation. Methods we did a diagnostic and excisional biopsy of the pigmentated lesion Result, as a result of the biopsy, we found that amalgam tattoo with a granulomatous reaction to a foreign body. Conclusion: By means of an excisional biopsy, the diagnosis of the lesion observed in the patient was verified, thus resulting in an amalgam tattoo with a granulomatous reaction to a foreign body.
\end{abstract}

Keywords: Dental Amalgams, tattooing, argentum metallicum, pigmentation disorders, Macula 


\section{Resumen}

El tatuaje de amalgama o argirosis focal, es una lesión iatrogénica que sigue a la implantación traumática de partículas de amalgama en tejido blando o a la transferencia pasiva por fricción crónica de la mucosa contra una restauración de amalgama se caracteriza por el depósito de restos de material restaurativo compuesto por una mezcla de plata, mercurio, zinc, estaño y cobre en el tejido conectivo. Objetivo presentar el caso clínico de un paciente con tatuaje por amalgama y el tratamiento que fue realizado previo a su rehabilitación dental. Métodos Se realizó diagnóstico y la biopsia excisional de la lesión pigmentada Resultado como resultado de la biopsia encontramos tatuaje por amalgama con reacción granulomatosa a cuerpo extraño. Conclusión: Por medio de una Biopsia excisional se comprobó el diagnóstico de la lesión que se observó en la paciente, resultando así un tatuaje por amalgama con reacción granulomatosa a cuerpo extraño

Palabras Clave: Amalgama dental, tatuaje, argentum metallicum, trastornos de la pigmentación, Macula.

\section{INTRODUCCIÓN}

Las lesiones pigmentadas de la cavidad oral son infrecuentes y pueden tener un origen melanocítico o no melanocítico. Pueden clasificarse clínicamente en pigmentaciones focales como mácula melanótica oral, tatuaje de amalgama, nevo melanocítico, melanoacantoma, tumor neuroectodérmico melanótico de la infancia, melanoma y pigmentaciones multifocales o difusas, incluidas entidades como la pigmentación fisiológica (racial), hiperpigmentación inducida por fármacos, melanosis asociada al tabaquismo, pigmentación posinflamatoria, pigmentación de metales pesados y melanosis asociada con enfermedades sistémicas como la enfermedad de Addison, neurofibromatosis, síndrome de Peutz-Jeghers, síndrome de McCune-Albright, síndrome del complejo de Carney y Bannayan -Ruvalcaba- Síndrome de Riley ${ }^{1}$.

La pigmentación de las mucosas puede ocurrir debido a la deposición de materiales extraños exógenos como amalgama dental, pigmento de tatuaje o grafito $^{2}$. El tatuaje de amalgama o argirosis focal, es una lesión iatrogénica que sigue a la implantación traumática de partículas de amalgama en tejido blando $o$ a la transferencia pasiva por fricción crónica de la mucosa contra una restauración de amalgama ${ }^{3}$ se caracteriza por el depósito de restos de material restaurativo compuesto por una mezcla de plata, mercurio, zinc, estaño y cobre en el tejido conectivo ${ }^{4}$.

La amalgama es uno de los materiales de restauración dentales más utilizados para empastes dentales. Es una aleación de plata, mercurio, estaño y trazas de otros metales. Se han reportado reacciones de hipersensibilidad y su uso ha sido prohibido en varios países. ${ }^{5}$

Por lo general, sigue a la extracción de dientes portadores de amalgama, ${ }^{3}$ cualquier superficie de la mucosa puede estar involucrada, pero los más frecuentes son: encía, mucosa alveolar y mucosa bucal ${ }^{6,7}$. Es la pigmentación más común de las membranas mucosas orales, ${ }^{3}$ aparecen como máculas ligeramente elevadas, de color negro, azul o gris, de bordes bien definidos, irregulares o difusos. Los bordes pueden tener un patrón homogéneo, ligeramente granulado. El color de la pigmentación oral puede variar según la cantidad y profundidad o ubicación del pigmento. La mayoría de los tatuajes de amalgama miden $6 \mathrm{~mm}$ o menos. Puede producirse un agrandamiento, presumiblemente debido a la migración de macrófagos, la reabsorción del hueso subyacente o la corrosión continua $^{2,5,8}$.

Las restauraciones de amalgama todavía se realizan en consultorios o clinicas de atendimiento dental por lo que la argirosis focal sigue siendo una de las causas más comunes de pigmentación intraoral y se debe considerar un diagnóstico diferencial importante cuando se evalúa a pacientes con sospecha de melanoma mucoso de la cavidad oral. La información sobre trabajos protésicos dentales previos debe incluirse en el historial médico del paciente, y una radiografía que muestre depósitos de metal en la mucosa puede descartar con seguridad un melanoma mucoso 9 .

El diagnóstico puede ser radiográfico cuando los fragmentos metálicos son lo suficientemente grandes para ser visibles como partículas radiopacas ${ }^{10}$. En caso de duda, se recomienda una biopsia diagnóstica para examen histopatológico 9 . El examen microscópico del 
tatuaje de la amalgama muestra numerosos gránulos negros finos dentro del tejido conectivo que muestran afinidad por las fibras de reticulina. Las lesiones extensas en la zona cosmética pueden tratarse con éxito con escisión quirúrgica e injerto gingival ${ }^{2}$.

El objetivo de este artículo es presentar el caso clínico de una paciente con tatuaje por amalgama con reacción granulomatosa a cuerpo extraño y el tratamiento que fue realizado previo a su rehabilitación dental.

\section{Reporte de caso}

Paciente femenina de 59 años, residente de Guadalajara, Jalisco; acudió a la Facultad de Odontología de la Universidad Autónoma de Guadalajara, con el interés de colocarse placas removibles. Se lee y firma el consentimiento informado para realización del tratamiento y se realiza la historia médica. A la exploración clínica se observa una mácula pigmentada de forma ovoide color gris obscura con bordes bien definidos que mide aproximadamente $3 \times 2 \mathrm{~mm}$, localizada en el reborde alveolar inferior izquierdo a nivel del primer molar (Fig.1A) extendiéndose de vestibular a lingual, (Fig.1B) con 7 años de evolución.

La paciente relató que la lesión apareció después de la extracción del premolar con restauración de amalgama, con crecimiento paulatino hasta llegar a tener el tamaño actual, no presentaba dolor. Se procedió a realizar toma radiográfica periapical en la cual se observaron pequeños fragmentos radiopacos ubicados en el reborde alveolar (Fig.2). Se procedió a realizar la biopsia excisional de la lesión bajo anestesia local (Lidocaína con epinefrina al 2\%) (Fig.3) y se envió para su análisis histopatológico en el departamento de Patología Bucal, reportando diagnóstico: "Argirosis focal con reacción granulomatosa a cuerpo extraño" (Fig.4). Posteriormente se derivó a la paciente al departamento de Restaurativa de la Universidad para la realización de las prótesis removibles y rehabilitación de las piezas dentales.

\section{Discusión}

El objetivo de este artículo fue presentar el caso clínico de una paciente con tatuaje por amalgama con reacción granulomatosa a cuerpo extraño, debido a que este tipo de lesiones se presentan por restos de amalgama que pueden impregnarse al hacerse la remoción de las mismas sin el uso de aislamiento absoluto.

Una amalgama dental es una aleación de mercurio, plata, cobre, estaño y que también puede contener paladio, zinc, y otros elementos para mejorar las características de manipulación y rendimiento clínico ${ }^{11}$. La amalgama ha sido utilizada por los dentistas por más de 100 años porque es muy durable y más accesible que otros materiales restaurativos dentales ${ }^{11}$, sin embargo, de hacerse necesaria la substitución o cambio de material, ésta debe realizarse bajo el uso de aislamiento absoluto, con lo que se reduce la cantidad de aspiración de aerosoles al paciente por vía oral y nasal, así como se evita que fragmentos de este material queden en la cavidad bucal y puedan producir este tipo de pigmentaciones, además es importante mencionar también el uso de equipo de protección para disminuir la exposición de aerosoles que sufren los odontólogos al vapor de mercurio o partículas de amalgama durante los procedimientos operatorios de remoción ${ }^{12}$. Las lesiones de pigmentación oscura de la mucosa oral pueden representar un desafío diagnóstico importante. Por lo general, se requiere una biopsia para determinar la naturaleza de tales decoloraciones intraorales ${ }^{13}$. Natarajan nos dice que el descubrimiento de lesiones mucosas de color negro o marrón en la región orofacial puede presentar un dilema para determinarlas. La mayoría de las lesiones pigmentadas que ocurren en las superficies mucosas de la región de la cabeza y el cuello son benignas y están asociadas con una serie de factores locales. En la región oral y perioral, la gran mayoría de las lesiones pigmentadas en esta área son máculas melanóticas orales y labiales, tatuajes de amalgama, seguidos de nevos melanocíticos orales y otras lesiones pigmentadas ${ }^{14}$.

Laimer y colaboradores nos mencionan que las lesiones pigmentadas de la mucosa oral son un hallazgo común ${ }^{13}$. La mayoría de las pigmentaciones son lesiones benignas como nevos, máculas melanóticas o tatuajes de amalgamas. Por el contrario, los melanomas de las mucosas son raros, pero a menudo letales; por lo tanto, excluir los melanomas orales en este contexto es crucial $^{15}$. Los factores exógenos, como los tatuajes de amalgama, representan una parte importante de estas lesiones. La mayoría de los tatuajes de amalgama se deben a tratamientos dentales cuando las partículas de metal se depositan 
accidentalmente en heridas orales abiertas o se estrellan como una metralla en la mucosa oral durante los procedimientos de preparación del diente $^{14}$

Rosebusch y colaboradores en 2019, nos comentan que la pigmentación de las mucosas puede ocurrir debido a la deposición de materiales exógenos en las mucosas ${ }^{2}$, mientras que otros autores señalan que puede ser llevada a cabo esta deposición por medio de la extracción de dientes con amalgama dental y que cualquier parte de la mucosa puede estar involucrada, mencionando que los sitios con mayor frecuencia son: la encía, la mucosa alveolar y la mucosa alveolar ${ }^{6,7}$.

Regezi $^{3}$ en su libro sobre Patología Oral señala que la importancia del tatuaje por amalgama radica en su similitud clínica con las lesiones productoras de melanina, las lesiones pigmentadas pueden diagnosticarse clínicamente según el tamaño, la forma o el color, junto con la información clínica y que desarrollo de un diagnóstico diferencial es imperativo para un médico que se enfrenta a estas lesiones para poder tratar adecuadamente al paciente como nos relata Gondak ${ }^{10}$. Neville y colaboradores ${ }^{4}$ se refieren a la necesidad de obtener radiografías en un intento de demostrar la presencia de los fragmentos metálicos y no ser requerido ningún tratamiento, demarcando que es por esto que es la pigmentación mas común de las membranas mucosas orales, por lo que Forsell y colaboradores ${ }^{10}$ nos recuerda la posibilidad de reacción a cuerpo extraño de los tejidos, junto con el cambio clínico de la lesión y la indicación de su remoción.

\section{Conclusión}

La presente lesión que se expone en este artículo tenía la peculiaridad de que la pigmentación era de vestibular a lingual sin presentar tinción en la parte superior del reborde alveolar por lo que se diferenciaba de cualquier otra lesión observada en otros pacientes por lo que es de gran importancia para el odontólogo, conocer las características clínicas principales del tatuaje por amalgama, como sus principales localizaciones y saber emplear métodos diagnósticos que nos permitan corroborar la presencia de partículas de metal alojados en el tejido para así poder descartar patología pigmentaria de mayor importancia como son los nevos celulares y el melanoma, es importante alertar al paciente de la existencia de este tipo de lesiones y realizar el tratamiento indicado previo a la rehabilitación dental que sea necesario en el caso clínico.

\section{Conflicto de intereses/Conflict of interest}

Todos los autores declaran que no existen conflictos potenciales de interés con respecto a la autoría y / o publicación de este artículo.

All authors declare no potential conflicts of interest with respect to the authorship and/or publication of this article.

\section{Referencias}

1. Albuquerque DMdS, Cunha JLS, Roza ALOC, Arboleda LPA, Santos- Silva AR, Lopes MA, et al. Oral pigmented lesions: a retrospective analysis from Brazil. Med Oral Patol Oral Cir Bucal. 2021;26(3):e284-91.

2. Rosebush MS, Briody AN, Cordell KG. Black and Brown: Non-neoplastic Pigmentation of the Oral Mucosa. Head Neck Pathol. 2019;13(1):47-55.

3. Regezi JA, Sciubba JJ, Jordan RCK, Oral Pathology: Clinical Pathologic Correlations, 7th ed. China: Elsevier, 2017.

4. Neville BW, Damm DD, Allen CM, Chi AC, Oral and Maxillofacial Pathology, 4th ed. Canada: Elsevier, 2016.

5. Arjona-Aguilera C, Collantes-Rodríguez C, GilJassogne C, Ossorio-García L, Jiménez-Gallo D. Pigmented oral lesion in a patient with metastatic melanoma. Indian J Dermatol Venereol Leprol. 2018 ;84(1):117-119.

6. Parizi J. Nai G. Amalgam tattoo: a cause of sinusitis?. Journal of Applied Oral Science. 2010;18(1):100-104.

7. GojkovVukelic M, Hadzic S, Pasic E. Laser Treatment of Oral Mucosa Tattoo. Acta Inform Med. 2011;19(4):244-246.

8. Gondak RO, da Silva-Jorge R, Jorge J, Lopes MA, Vargas PA. Oral pigmented lesions: Clinicopathologic features and review of the literature. Med Oral Patol Oral Cir Bucal. 2012;17(6):e919-24.

9. Lundin K, Schmidt G, Bonde C. Amalgam tattoo mimicking mucosal melanoma: a diagnostic dilemma revisited. Case Rep Dent. 2013;2013:787294.

10. Forsell M, Larsson B, Ljungqvist A, Carlmark B, Johansson O. Mercury content in amalgam tattoos of humanoral mucosa and its relation to local tissue reactions. Eur J Oral Sci. 1998;106(1):582-587.

11. Anusavice KJ. Philips' Science of Dental Materials: Elsevier 11th ed. Canada: Elsevier, 2003.

12. Vázquez ASJ, Sandoval SE, Chung R, García PMP, Cornejo VJA. Identification of Mercury Levels on the Operator's FaceMask During the Removal Of Dental 
Amalgamas: Pilot Study. Adv Dent \& Oral Health. 2017;3(3):55613.

13. Laimer J, Henn R, Helten T, Sprung S, Zelger B, Zelger B, Steiner R, Schnabl D, Offermanns V, Bruckmoser E, Huck CW. Amalgam tattoo versus melanocytic neoplasm - Differential diagnosis of dark pigmented oral mucosa lesions using infrared spectroscopy. PLoS One. 2018;13(11):e0207026.

14. Natarajan E. Black and Brown Oro-facial Mucocutaneous Neoplasms. Head Neck Pathol. 2019;13(1):56-70.

15. Yélamos O, Cordova M, Peterson G, Pulitzer MP, Singh B, Rajadhyaksha M, DeFazio JL. In vivo intraoral reflectance confocal microscopy of an amalgam tattoo. Dermatol Pract Concept. 2017;7(4):13-16.

\section{(ब) $\odot \odot$}

Publisher's Note: This article is an open access article distributed under the terms and conditions of the Creative Commons Attribution(CC BY) license (http://creativecommons.org/licenses/by/4.0/) 


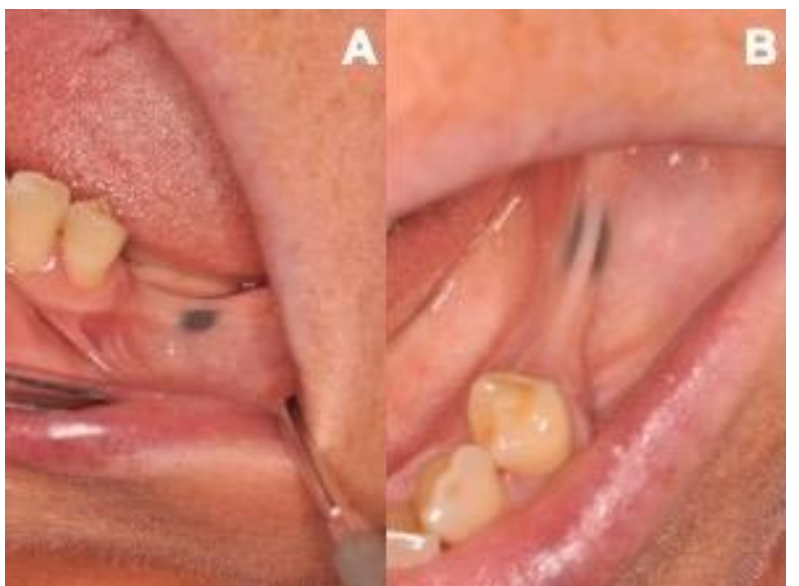

Figura 1: A: Vista vestibular de mácula; B: Vista oclusal en donde se aprecia extensión vestibulo lingual 


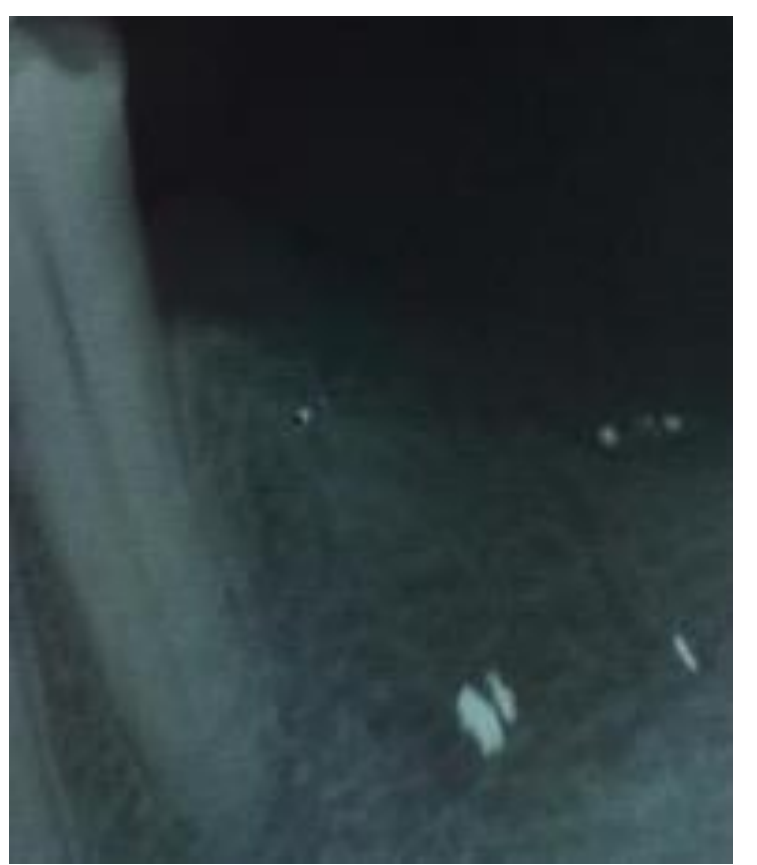

Figura 2: Rx. Periapical con presencia de fragmentos radiopacos. 


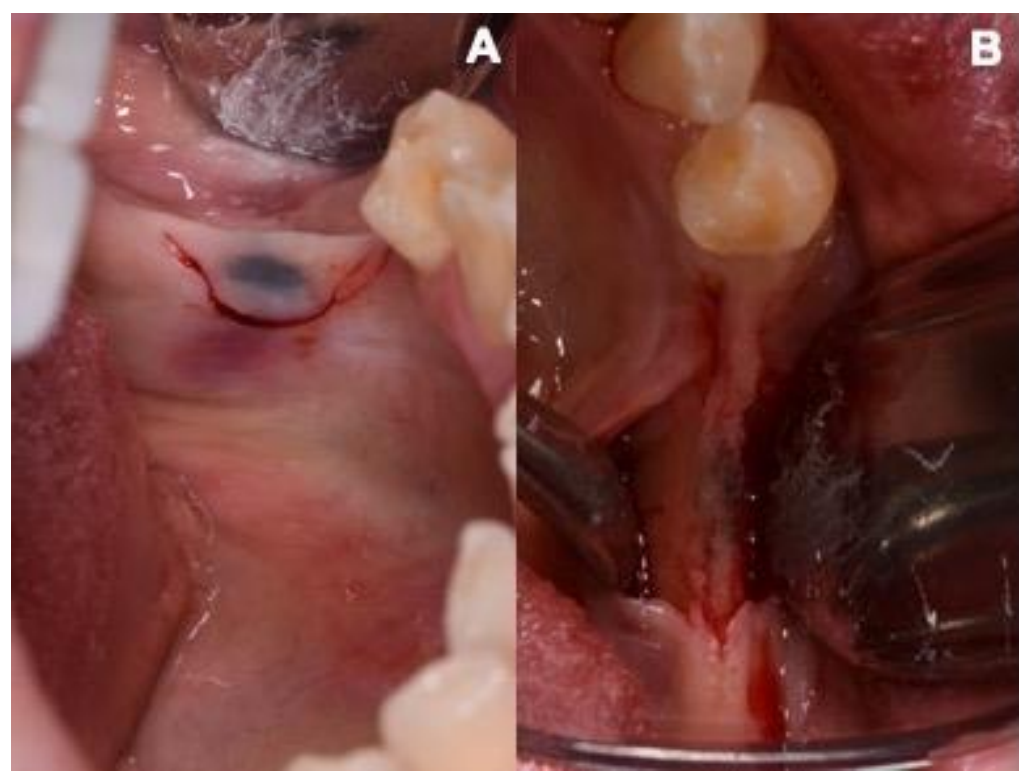

Figura 3: A: Incisión para la toma de biopsia excisional, B: Proceso alveolar pigmentado se respeta por la disminución de su altura. 


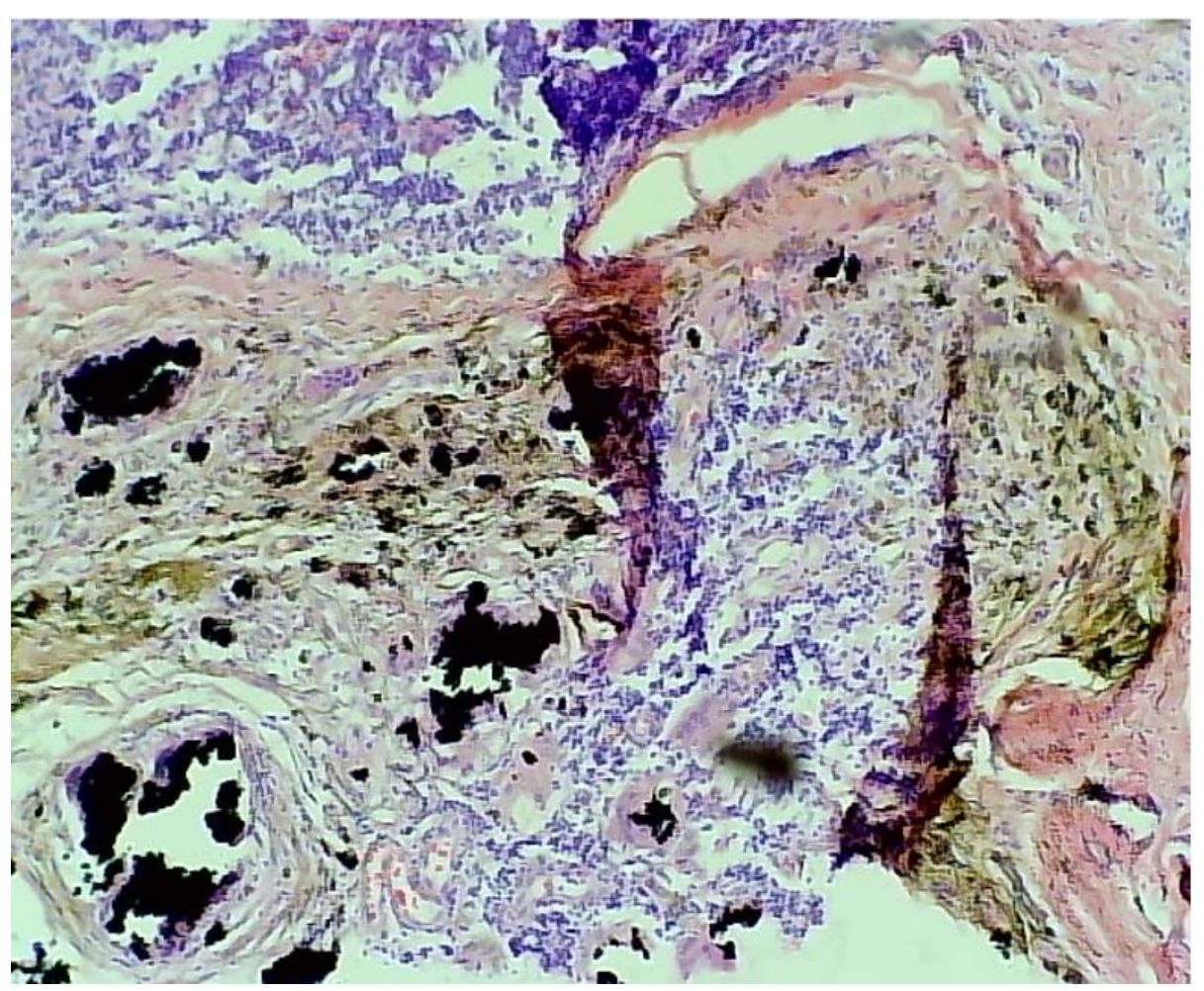

Figura 4: Corte histológico 40x que muestra material negruzco acelular promocionando reacción granulomatosa con células gigantes multinucleadas a cuerpo extrañ 